\title{
The Technology and Setup for High-Throughput Synthesis of Endohedral Metal Fullerenes
}

\author{
D.I. Chervyakova, G.N. Churilov, A.I. Dudnik, G.A. Glushenko, \\ E.A. Kovaleva, A.A. Kuzubov, N.S. Nikolaev, I.V. Osipova \\ and N.G. Vnukova
}

\begin{abstract}
The article presented the technology and setup for high-throughput synthesis of carbon nanostructures. It was shown that the plasma-chemical synthesis of fullerenes and endohedral metallofullerenes (EMF) in high-frequency arc discharge can be controlled by changing helium pressure in the chamber. The methods of extraction using Lewis acids and separation of individual EMF by HPLC were suggested as the most effective.
\end{abstract}

Keywords Endohedral metallofullerenes • High-frequency arc discharge Pressure

D.I. Chervyakova E.A. Kovaleva · A.A. Kuzubov · N.S. Nikolaev

Siberian Federal University, Krasnoyarsk, Russia

e-mail: asteralin@gmail.com

E.A. Kovaleva

e-mail: kovaleva.evgeniya1991@mail.ru

A.A. Kuzubov

e-mail: alexxkuzubov@gmail.com

N.S. Nikolaev

e-mail: churilov@iph.krasn.ru

G.N. Churilov ( $\square)$ · A.I. Dudnik · G.A. Glushenko · I.V. Osipova · N.G. Vnukova

Kirensky Institute of Physics Federal Research Center KSC Siberian Branch Russian Academy of Sciences, Krasnoyarsk, Russia

e-mail: churilov@iph.krasn.ru
A.I. Dudnik
e-mail: churilov@iph.krasn.ru
G.A. Glushenko
e-mail: churilov@iph.krasn.ru

I.V. Osipova

e-mail: churilov@iph.krasn.ru

N.G. Vnukova

e-mail: churilov@iph.krasn.ru

(C) The Author(s) 2018 


\section{Introduction}

Endohedral metallofullerenes (EMF) have stimulated great scientific interest [1]. From the fundamental point of view, this is because they engender new spherical molecules with structure which is similar to the electronic structure of the atom. Indeed, there is a positively charged nucleus - a metal ion and the excess electron density at a quasi-spherical surface, where the carbon atoms have a generalized $\pi$-system. EMF offers a broad range of properties that make them appealing for use in different fields, such as material science and nanomedicine [2]. Already EMF-base substances are reagents for medicinal drugs and contrast agents for magnetic imaging. In the future, due to the unique properties, we can expect an even greater requirement of EMF, than is available today. This requires considerably reduce the cost of producing EMF, i.e., develop more efficient technologies of synthesis and isolation.

This can be done on the basis of the available experimental results and theoretical concepts that define the model representation assembly of molecules and the formation of substances in powder form. We believe that there are the following most important theoretical concepts defining the process of formation of EMF:

1. Fullerenes are formed through a process of clustering from $\mathrm{C}_{2}$ [3].

2. First, large clusters are produced. That clusters stabilized in shape and size through the loss of $\mathrm{C}_{2}$. This is described by "shrinking hot giant fullerene" concept [4].

The most important experimental results:

3. Effective synthesis of fullerene and EMF may be only in a helium atmosphere.

4. Plasma must be carbon arc.

5. EMF, as well as fullerenes, are formed during quenching in the cooling of the plasma layer, wherein the temperature gradient, and for arc plasma and the electron density, have the most important control value [5].

6. EMF extraction must be in a Soxhlet apparatus, and concentration by using Lewis acids.

7. Separation of individual EMF may be by HPLC.

How effectively we have answered these questions, so will be our efficient method of producing EMF.

\section{Kinetic Study of Metallofullerene Formation}

It is well known that fullerenes are made of $\mathrm{sp}^{2}$ hybridized atoms. We report the results of QM/MD simulations leading to completely closed cage fullerene structures and identify key steps during these dynamics (Fig. 1). 


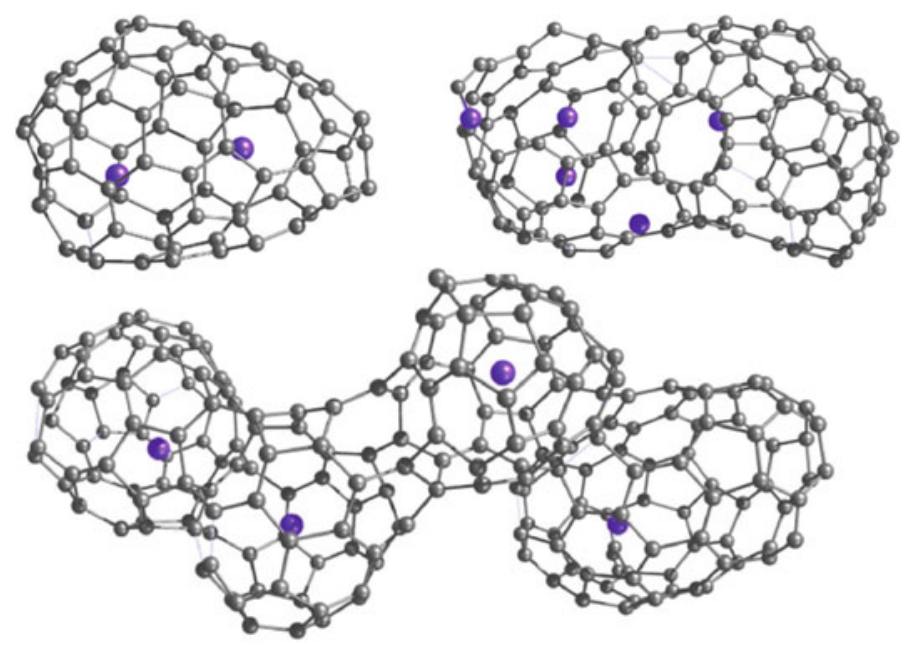

Fig. 1 Giant metallofullerene structures obtained during the simulation

The process of fullerene formation can be considered as a three-step process: $\mathrm{C}_{2}$ units coalescence leads to the formation of chains, chains form $\mathrm{sp}^{2}$ structures, and finally, $\mathrm{sp}^{2}$ structures bend in order to form the cage.

We suggested that the first step follows second-order kinetics while the third step is first-order chemical reaction and studied the dependence of the rate constant for them as a function of temperature and He concentration.

Rate of the chain formation increases rapidly up to $1800 \mathrm{~K}$ due to the larger number of effective $\mathrm{C}_{2}$ collisions. Further increasing of the temperature leads to the break of the bonds and thus lowers the rate constant. The same effect is observed for the cage formation.

Helium atoms act as thermostat taking the extra energy from forming carbon chains and thus helping the formation of larger chains and $\mathrm{sp}^{2}$ structures. However, as the number of $\mathrm{He}$ atoms in $100 * 100 * 100 \AA$ box reaches 150 , they start to "freeze" $\mathrm{sp}^{2}$ fragments decreasing the possibility of regrouping barrier overcoming.

\section{Description of the Experiment}

\section{Method of EMF Synthesis}

Our setup, as well as other setups of this type, is based on the process of cooling the carbonaceous plasma in a helium atmosphere. The plasma is obtained as a result of arc discharge evaporation of graphite rods, or graphite rods are filled with additional substances - dopants [5, 6], i.e., the operation of the setup is based on the W. Kraetchmer's modified method. The distinguished feature of the proposed setup, compared to most of the fullerene generators used nowadays, is the 
application of medium frequency alternate-current arc. Symmetrical arrangement of the electrodes allows to reach up to a $100 \%$ conversion of the electrode material into the fullerenes and EMF containing $\mathrm{CC}[7,8]$. As a consequence, this allows to avoid the main disadvantage typical for the setups operating on a direct current. The parameters of synthesis can be varied within the wide range of limits: arc current from 50 to $400 \mathrm{~A}$, current frequency from 20 to $160 \mathrm{kHz}$, the helium pressure in the chamber from 30 to $400 \mathrm{kPa}$. This broad variability of parameters allows finding optimal values not only for the regular fullerene, but also for the EMF. The chamber, in which the synthesis is performed, is equipped with quartz windows for visual and spectral studies when the setup is combined with the spectrograph.

The profound increase in the setup capacity combined with the enlarged variety of combination options results into a visibly increased productivity of $\mathrm{CC}$ along with the high amount of fullerenes and EMF as well to obtain renewed experimental results revealing the degree of influence of various synthesis parameters upon the process of various type EMF generation.

Figure 2 demonstrates the block scheme of the setup. The chamber, in which the synthesis is performed, is cooled by water. Two pairs of graphite electrodes placed on water-cooled rods on the ends are evenly placed into the chamber. The feeding of each rod is made by a special mechanical drive, activated by the stepping motor connected to the control unit. The control unit adjusts the flow rate of the rods so that the arc current remains constant corresponding to a predetermined initial current in the range of $2-3 \%$.

The pressure of the helium, which is fed directly from the cylinder through a pressure regulator and a nitrogen trap into the chamber, is also kept constant, with a

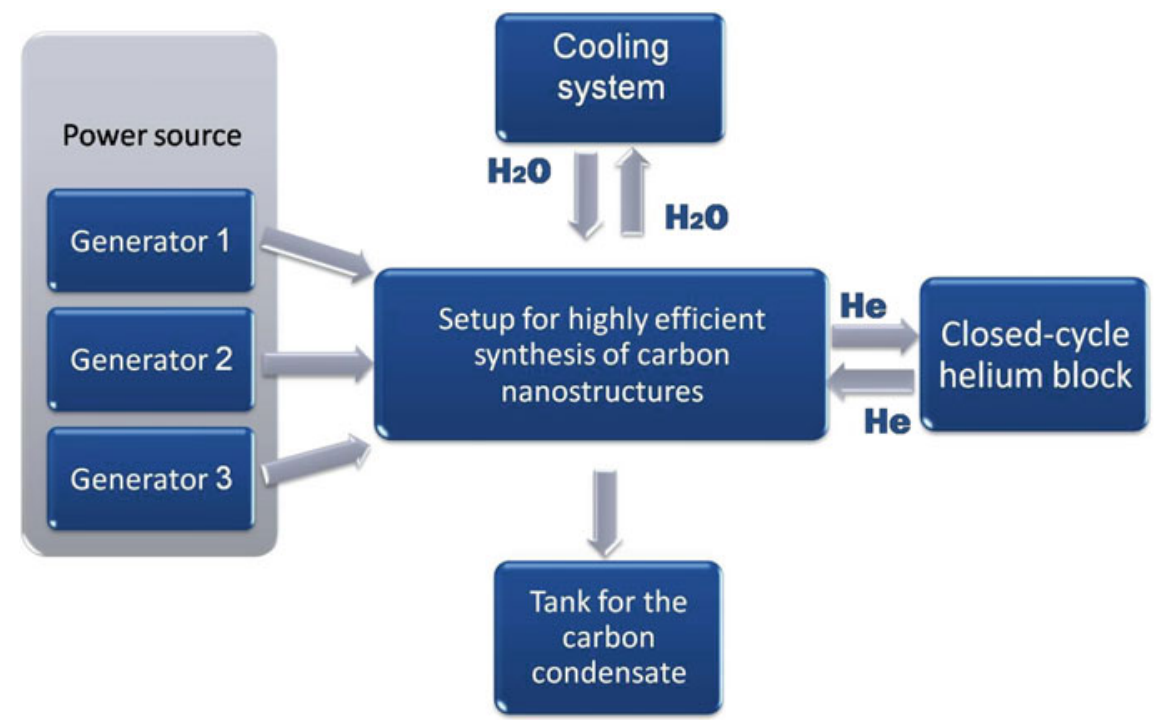

Fig. 2 Block scheme of the setup for the synthesis of $\mathrm{CC}$ 
special device, made on the basis of a controlled nonreturn valve and matching to given number, in the range of 2-3\%. Power of each pair of electrodes is carried out by a separate generator $\mathrm{G} 1$ and $\mathrm{G} 2,25 \mathrm{~kW}$, via the matching blocks. Figure 3 shows a circuit diagram of the installation, in which we can see that a matching unit consists of two reducing transformers $\mathrm{T} 1$ and $\mathrm{T} 2$ with a transformation ratio of 3:1. The secondary windings of transformers are connected sequentially to two capacitors $\mathrm{C} 1$ and $\mathrm{C} 2$, an inductance coil L1, a ballast resistor R1, and two graphite electrodes with the arc in a gap between them. Another matching unit also consists of two reducing transformers $\mathrm{T} 3$ and $\mathrm{T} 4$ with a transformer ratio $3: 1$ and an analogous set of capacitors (C3 and C4), resistor (R2), inductive coil (L2) and another pair of graphite electrodes. The voltage of current transformer TA1 and TA2 is supplied to the unit controlling the feed rate [9].

Synthesis of nano-dispersed substances based on carbon, performed on setup developed in this work, is possible using graphite rods with a diameter of 4-20 mm. Rods of $6 \mathrm{~mm}$ in diameter and $100 \mathrm{~mm}$ long were evaporated. The rods were annealed preliminarily in a water-cooled chamber at $10 \mathrm{~Pa}$, at a temperature of $1800-2000 \mathrm{~K}$ for $30 \mathrm{~min}$. The same rods, only predrilled along the central axis, were used for the EMF synthesis. A hole with a diameter of $3 \mathrm{~mm}$ and a length of $85 \mathrm{~mm}$ was filled with a mixture of graphite powder and metal oxide in mass ratio of 1:1. Annealing was performed under the same conditions as those used in the preparation of the rods to obtain conventional fullerenes.

Prior to the synthesis, the chamber is warmed up with the water heated to a temperature of $60-70{ }^{\circ} \mathrm{C}$ during $15 \mathrm{~min}$. Then through the top entrance nitrogen trap, the chamber is purged with helium for 1-2 min at the speed of 4-6 1/min. During the synthesis process, the chamber is cooled by the cold water with a

Fig. 3 Electrical scheme of the setup

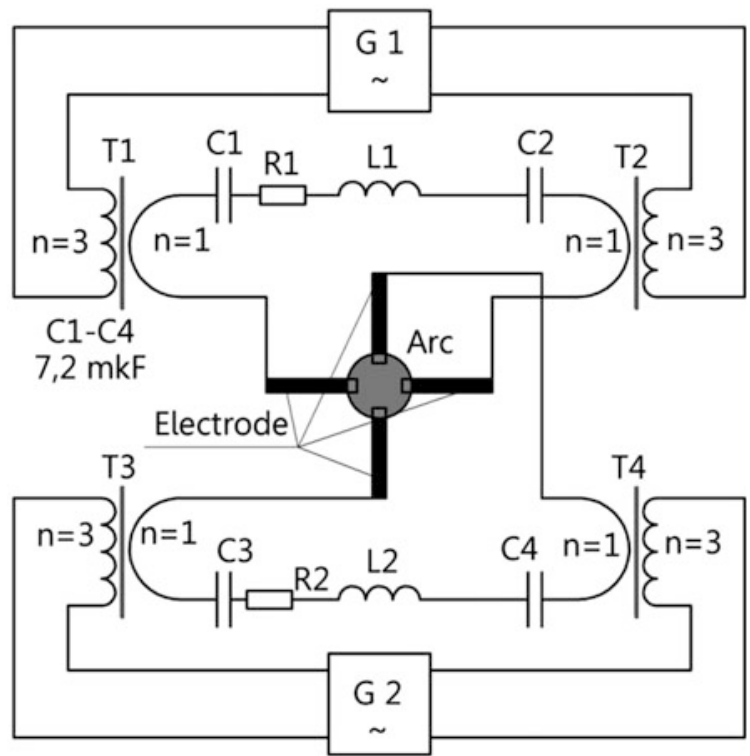


temperature of $10-30{ }^{\circ} \mathrm{C}$, and helium is supplied at a rate of $91 / \mathrm{min}$ through the flowmeter and pressure regulator, directly from the cylinder container. Cooling of the trap is carried out by liquid nitrogen.

Fullerene content was estimated by atom emission analysis. Atomic emission analysis was performed on the setup, consisting of plasma atomizer-HF discharge in a stream of argon with copper and graphite electrodes, the spectrograph PGS-2 and a computerized spectral registration [10]. The mass spectra of sample were recorded by Bruker Autoflex time of flight mass spectrometer with laser desorption. To optimize the condition of synthesis, we studied the influence of the current range and frequency and helium gas pressure in the chamber on the structure of the formed CC and the content of different fullerenes and EMF. This analysis showed that within the limits of parameters available for our setup, variation of the current parameters and gas pressure lead to the same results. In other words, the structure of the $\mathrm{CC}$, the quantitative content of the fullerenes and $\mathrm{EMF}$ in the $\mathrm{CC}$, and their composition depend largely on the pressure. All the changes that can be obtained changing the current and the frequency can be also obtained by changing the pressure in the chamber. Mass spectrum of fullerene, synthesized with the addition of $\mathrm{Gd}_{2} \mathrm{O}_{3}$ under chamber pressure 64.8, is presented in Fig. 4 [11].

\section{Method of EMF Extraction}

Fullerenes were extracted by $\mathrm{CS}_{2}$ in a Soxhlet apparatus. The fullerene solution was evaporated and the fullerene yield was determined by the weight ratio of fullerite and soot (prior to extraction). In order to enrich of FM with EMF, the complexation

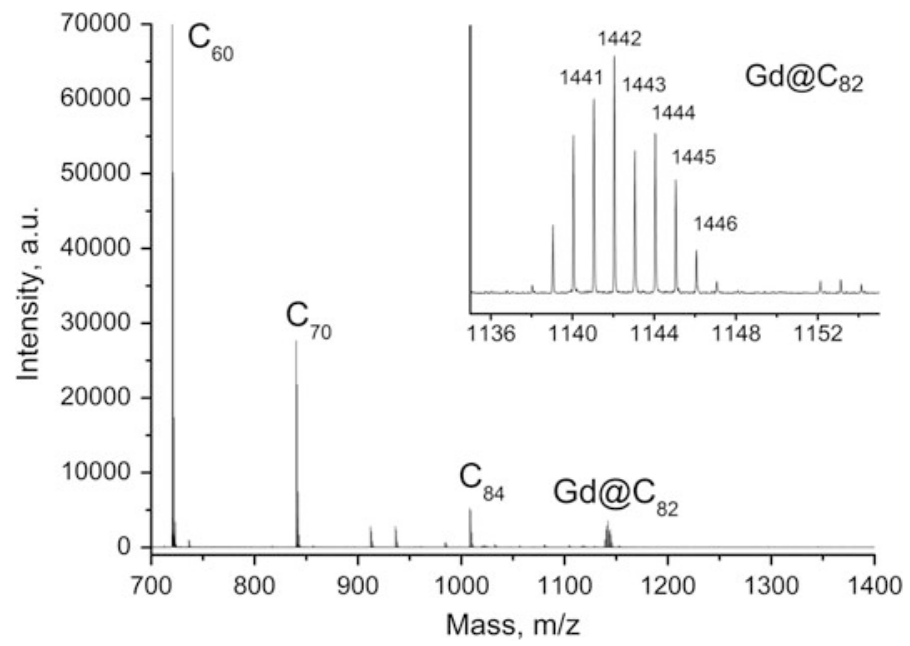

Fig. 4 Mass spectral (positive ion) analysis of fullerenes, detached from CC synthesis under 64.8 chamber pressure 
reaction with Lewis acids was used $[12,13]$. The resulting filtrate is a mixture of EMF and empty fullerenes, which accounted for most of the EMF. Isolation of individual EMF was carried out by high-performance liquid chromatography (HPLC). It should be noted that $\mathrm{TiCl}_{4}$ Lewis acid is most effective and powerful in purification for the various types of pure metallofullerenes. Other Lewis acids $\left(\mathrm{CuCl}_{2}, \mathrm{AlCl}_{3}\right)$ did not demonstrate such results.

\section{Method of Quantitative Analysis of EMF}

The technique for rapid determination of endohedral metallofullerene (EMF) content in a fullerene mixture (FM) was used by [14]. Samples of the CC with Me (for example Gd, Y) obtained at pressures of 353, 225, 98, 64.8, and $32.4 \mathrm{kPa}$ were subject to extraction. The extracts were dried, weighted, and then dissolved in the initial solvent to the concentration of $1 \mathrm{mg} / \mathrm{mL}$. Sample solutions were placed into the holes in the graphite rods and then atomized in the arc discharge in a setup for emission analysis. Standard samples were prepared beforehand using solutions with known Gd concentration in the holes in the graphite rods. Obtained data were used for building the concentration curve for analysis using standard samples [15]. For example, gadolinium content in a given probe was found from the intensity of its line at $\lambda=335.86 \mathrm{~nm}$ and the concentration curve. Allowing for the mass spectrum showed that gadolinium is contained only in $\mathrm{Gd} @ \mathrm{C}_{82}$, we thus measure mass fraction of this compound in the fullerene extract. Results of investigation of the EMF content in the fullerene mixture extracted from the CC at various pressures are given in Table 1.

Table 1 Dependence of the content of EMF in the FM extracted from the CC obtained under various helium pressures

\begin{tabular}{l|l|l|l|l|l}
\hline Helium pressure in chamber, $\mathrm{kPa}$ & \multicolumn{6}{l}{$l$} & \\
\hline 360 & 120 & 60 & & \\
\hline \\
Extraction
\end{tabular}




\section{Conclusion}

A method for high-throughput synthesis of carbon nanostructures in a high-frequency arc discharge in the flow of helium was presented. It was shown that the plasma-chemical synthesis of fullerenes and EMF in high-frequency arc discharge can be controlled by changing helium pressure in the chamber. The results may also form the basis of the concepts of formation EMF.

Acknowledgements Research is carried out with the financial support of the state represented by the Ministry of Education and Science of the Russian Federation. Agreement no. 14.613.21.0010, 27 Aug 2014. Unique project Identifier: RFMEFI61314X0010.

\section{References}

1. Popov, A.A., Yang, S., Dunsch, L.: Endohedral fullerenes. Chem. Rev. 113, 5989-6113 (2013)

2. Yang, S., Lui, F., Chen, C., Jiao, M., Wei, T.: Fullerenes encaging metal clustersClusterfullerenes. Chem. Commun. 47, 11822-11839 (2011)

3. Afanas'ev, D.V., Bogdanov, A.A., Dyuzhev, G.A., Kruglikov, A.A.: Formation of fullerenes in an arc discharge. Tech. Phys. 42, 234-241 (1997)

4. Irle, S., Zheng, G., Wang, Z., Morokuma, K.: The $\mathrm{C}_{60}$ formation puzzle "Solved": QM/MD simulations reveal the shrinking hot giant road of the dynamic fullerene self-assembly mechanism. J. Chem. Phys. 110, 14531-14576 (2006)

5. Churilov, G.N., Vnukova, N.G.: The method of synthesis of endohedral fullerenes. Patent. 2582697

6. Churilov, G.N.: Synthesis of fullerenes and other nanomaterials in arc discharge. Fullerenes, Nanotubes Carbon Nanostruct. 16, 395-403 (2008)

7. Bezmelnitsyn, V., Davis, S., Zhou, Z. Efficient synthesis of endohedral metallofullerenes in 3-phase arc discharge. Fullerenes, Nanotubes Carbon Nanostruct. 23. 612-617 (2014)

8. Churilov, G.N., Kratschmer, W., Osipova, I.V., Glushenko, G.A., Vnukova, N.G., Kolonenko, A.L., Dudnik, A.I. Synthesis of fullerenes in a high-frequency arc plasma under elevated helium pressure. Carbon. 62, 389-392 (2013)

9. Churilov, G.N., Popov, A.A., Vnukova, N.G., Dudnik, A.I., Samoylova, N.A., Glushenko, G. A.: Controlled synthesis of fullerenes and endohedral metallofullerenes in high frequency arc discharge. Fullerenes, Nanotubes Carbon Nanostruct. 24(11), 675-678 (2016)

10. Sichenko, D.P., Vnukova, N.G., Lopatin, V.A., Glushchenko, G.A., Marachevskiy, A.V., Churilov, G.N.: A facility for atomic emission spectral analysis and methods for spectrum processing. Instrum. Exp. Tech. 47(4), 489-492 (2004)

11. Churilov, G.N., Popov, A.A., Vnukova, N.G. Dudnik, A.I., Glushchenko, G.A., Samoilova, N.A., Dubinina, I.A., Gulyaeva, U.E.: Method and setup for high-controlled synthesis of fullerenes and endohedral Metallofullerenes. JETP Lett. 9, 64-70 (2016)

12. Akiyama, K., Hamano, T., Nakanishi, Y., Takeuchi, E., Noda, S., Wang, Z., Kubuki, S., Shinohara, H.: Non-HPLC rapid separation of metallofullerenes and empty cages with $\mathrm{TiCl}_{4}$ Lewis Acid. J. Am. Chem. Soc. 134, 9762-9767 (2012)

13. Stevenson, S., Rottinger, K.A., Fahim, M., Field, J.S., Martin, B.R., Arvola, K.D.: Inorg. Chem. 53, 12939-12946 (2014) 
14. Churilov, G.N., Popov, A.A., Guliaeva, U.E., Samoylova, N.A., Vnukova, N.G., Kolonenko, A.L., Isakova, V.G., Dudnik, A.I., Koravanets, V.S.: Express analysis of endohedral fullerenes amount contained at fullerene mixture. Nanosystems: physics, chemistry, mathematics. 7, 140-145 (2016)

15. Torok, T., Mika, J., Gegus, E.: Emission Spectrochemical Analysis. Akademia Kiado, Budapest (1978); Mir, Moscow (1982)

Open Access This chapter is licensed under the terms of the Creative Commons Attribution 4.0 International License (http://creativecommons.org/licenses/by/4.0/), which permits use, sharing, adaptation, distribution and reproduction in any medium or format, as long as you give appropriate credit to the original author(s) and the source, provide a link to the Creative Commons license and indicate if changes were made.

The images or other third party material in this chapter are included in the chapter's Creative Commons license, unless indicated otherwise in a credit line to the material. If material is not included in the chapter's Creative Commons license and your intended use is not permitted by statutory regulation or exceeds the permitted use, you will need to obtain permission directly from the copyright holder. 